\title{
A discrete-trials/fixed-interval method of discrimination training*
}

\author{
W. T. WOODARD and M. E. BITTERMAN \\ University of Hawaii, Honolulu, Hawaii 96822
}

\begin{abstract}
An efficient new method of discrimination training is described which has several advantages over older free-operant and discrete-trials procedures. Illustrative data on reversal learning in pigeons and goldfish are presented.
\end{abstract}

In the conventional free-operant method of discrimination training, two stimuli are presented in alternating sequence for fixed periods of time-response to one $(\mathrm{S}+)$ intermittently reinforced (usually on a VI schedule), response to the other $\left(S_{-}\right)$never reinforced-and the frequency (or rate) of response to each stimulus is measured. The danger here is that, when the schedule of reinforcement is relatively dense, much of the responding in the presence of $\mathrm{S}+$ will be elicited, not by $S+$, but by the reinforcement itself (or some trace of it), which may serve as a discriminative stimulus. Control tests with goldfish, in fact, show significant "discrimination" between two identical stimuli under such conditions (Tennant \& Bitterman, 1973). While this pseudodiscrimination effect may be minimized by the use of a low-density schedule of reinforcement, the efficiency of the training procedure tends to be reduced at the same time. The only way entirely to eliminate the possibility that reinforcement will serve as a discriminative stimulus is to introduce test trials on which response to $\mathrm{S}+$ is not reinforced, a procedure which is unsatisfactory for two reasons: (1) where training trials do not themselves provide suitable measures of performance, we do not get a continuous picture of the course of discrimination, and (2) the test trials tend to wash out the effects of training, since the response contingencies are not the same in testing as in training.

In the conventional discrete-trials method of discrimination training, each presentation of $\mathrm{S}+$ terminates with the first reinforcement. Since S+ and Sare scheduled in quasirandom order, reinforcement (or failure of reinforcement) for response to one stimulus provides no information about probability of reinforcement for response to the succeeding stimulus, and fine-grain, trial-by-trial analysis of the course of discrimination becomes possible. With low-effort manipulative responses, such as leverpressing in rats, keypecking in pigeons, or target striking in fishes, the adequacy of performance depends first of all on the number of responses to $\mathrm{S}+$ required for reinforcement

*This work was supported by Grants MH 23294 and NS 06890 from the Public Health Service. Requests for reprints may be addressed to either author at the Laboratory of Sensory Sciences, 1993 East-West Road, Honolulu, Hawaii 96822. on each trial. If the first response to $\mathrm{S}+$ is reinforced, discrimination, as measured in terms of differential latency or probability of response, may be poor even with stimuli which are readily discriminated under other conditions. If, however, a ratio requirement is introduced (for example, the 10th leverpress in the presence of $\mathrm{S}+$ is reinforced), discrimination between the two stimuli soon is evidenced in terms of the time required to fulfill the ratio requirement (Graf, Bullock, \& Bitterman, 1964; Behrend, Bauman, \& Bitterman, 1965). This discrete-trials/fixed-ratio method is quite useful on the whole (see, for example, Woodard, Schoel, \& Bitterman, 1971), but time measures are not as satisfactory as frequency measures for two reasons: (1) they are not as easy to take, and (2) since their distribution is skewed by the occurrence of extreme scores (despite the time limit that must be used on each trial in order to keep session times within reasonable bounds), they require some sort of normalizing transformation. The method suffers also from the arbitrariness associated with the choice of ratio and time limit.

The advantages of both the older methods-free-operant/variable-interval and discretetrials/fixed-ratio-are combined in what may be called the discrete-trials/variable-interval method. The procedure is like that in free-operant/variable-interval training, except that each presentation of $S+$ terminates with the first reinforcement; $S$ - presentations are of comparably brief duration; and the two stimuli, separated by brief time-outs, follow each other in quasirandom orders. The possibility that reinforcement will serve as a discriminative stimulus is eliminated and a frequency measure, rather than a time measure, is employed. If the total durations of $S^{+}$and $S_{-}$in each session are equal, total frequencies of response to the two stimuli can be compared directly, or, if their durations are unequal, the comparison can be made in terms of rate. Where a trial-by-trial picture of the progress of the discrimination is desired, of course, conversion to rate is essential, since (in the VI case) stimulus duration changes from trial to trial. The chief disadvantage of this method, which we have tried both with pigeons and with goldfish, stems from the fact that response rate varies markedly with stimulus duration 
DAY 1

DAY 2

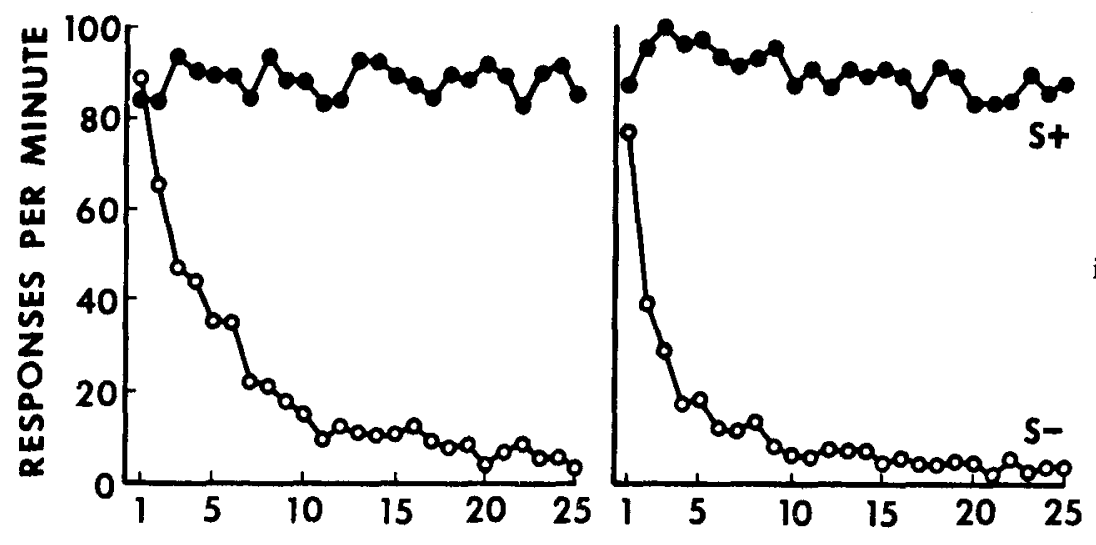

TRIALS

(for example, the $\mathrm{S}+$ rate for a $20-\mathrm{sec}$ presentation is much different from the rate for a 110 -sec presentation); to be able to plot a meaningful trial-by-trial learning curve, therefore, requires balancing of stimulus durations over animals. If, for example, only five different intervals are used, and if stimulus orders as well as intervals are to be balanced, the number of different conditions becomes rather large and the balancing procedure rather complex.

A much simpler alternative, from the point of view both of programming and of experimental design, is the discrete-trials/fixed-interval method. We had little hope for the method (and were reluctant even to try it) on the assumption that, in naive animals, the development of the discrimination between $\mathrm{S}+$ and $\mathrm{S}-$ would be confounded with the development of a temporal discrimination, and that, in experienced animals, the already developed temporal discrimination would reduce frequency of response to both stimuli and thus reduce the sensitivity of measurement. When, at length, we did look at performance with fixed intervals of $20 \mathrm{sec}$ in pigeons and goldfish, we were surprised to find little evidence of temporal discrimination, even after prolonged training. Visual discriminations are, however, established and reversed very rapidly by this method, as the sample data will show. We have, then, in discrete-trials/fixed-interval training, a highly efficient method which permits detailed analysis of rapidly changing performance in terms of the convenient and statistically acceptable frequency measure, with freedom from the concern that reinforcement may operate as a discriminative stimulus.

\section{SAMPLE DATA FOR PIGEONS}

\section{Method}

The Ss were eight male homing pigeons reduced to $80 \%$ of ad lib weight. They were trained in a ventilated picnic chest set into a larger, sound-reduced enclosure. Centered on one wall of the animal's compartment was a single pecking key which could be illuminated by colored lights, and directly below it was a feeder. The feeder was a grain-filled motor-driven box, normally retracted from the animal's compartment, which could be inserted into the compartment for a period of $2.5 \mathrm{sec}$ (during which it was illuminated with white light) and then retracted. All events of the experiment were controlled by conventional relay modules, and responses were recorded automatically on a printing counter.

The pigeons first were feeder-trained, autoshaped to peck a white key, and then trained in a series of 1- and 2-day discrimination reversals with a variety of stimuli and by a variety of methods approximating the one on which we finally settled. The method is the following: Each trial begins after a brief intertrial interval in darkness with illumination of the key by $\mathrm{S}+$ or $S$-. All responses are counted for a period of $20 \mathrm{sec}$ (the fixed interval), at the end of which a relay is locked up, and counting stops, but the key light stays on. In $\mathrm{S}^{+}$, the next response produces reinforcement-the key light is turned off and the feeder is presented-after which the next intertrial interval begins. In $S-$, the lock-up starts a 10 -sec penalty timer, which is reset by each subsequent response, and the trial ends only when the penalty timer times out; response to $S$ - thus is penalized by postponing its termination (Schoel, Davis, \& Bitterman, 1971). The orders of stimulus presentation are such that $S+$ and $S-$ appear equally often for each animal in each session, with no more than three $S+$ or $S-$ trials in succession. In any session, one of the two stimuli is $S+$ for four of the animals and $S-$ for the others. On any given trial, $S+$ is presented to four of the animals and $S \ldots$ to the others. In any given pair of trials, the order of stimuli is $\mathrm{S}+\mathrm{S}+$ for two animals, $\mathrm{S}+\mathrm{S}-$ for two animals, $\mathrm{S}-\mathrm{S}+$ for two animals, and $\mathbf{S}-\mathrm{S}-$ for the remaining two animals. This procedures makes it possible to plot perfectly balanced trial-by-trial learning curves for each session. The results to be considered are curves showing pooled within-sessions asymptotic reversal performance. (Since our purpose is simply to demonstrate the efficacy of the discrimination procedure, there will be no discussion of the mechanisms presumed to be responsible for discrimination reversal, and we deliberately refrain from presenting results for pigeons and goldfish obtained in comparable experiments in order not to become involved here with the problem of differences in the performance of the two species.)

\section{Results and Discussion}

Asymptotic performance of the pigeons trained to discriminate orange (Wratten No. 21) from violet (Wratten No. 34A) in a series of daily 50-trial sessions 
with positive and negative stimuli reversed every 2 days is shown in Fig. 1. The curves, plotted in terms of responses per minute, are based on the data of Reversals 9.16, over which analyses of variance showed that there was no significant change in performance. These results are strikingly similar to those obtained in discrete-trial/fixed-ratio experiments with pigeons (Woodard, Schoel, \& Bitterman, 1971, cf. their Fig. 2): S+ performance shows very little change, either within or between sessions, while $\mathrm{S}-$ performance, which is comparable to the St performance at the start of each session, quickly declines within sessions to a near zero level, the decline being slightly more precipitous on Day 2 than on Day 1. Even the number of $S$ - trials required to reach asymptote on each day is very much the same as in the fixed-ratio procedure. The fixed-interval procedure simply is more convenient.

While the use of fixed intervals immediately suggests the possibility of temporal discrimination, no such discrimination became evident. There was no change in mean scores, even after long series of reversals. Furthermore, breakdowns of response rate by time blocks within the 20-sec stimulus presentations give little evidence of temporal discrimination. A sample of such an analysis, based on all trials of one of the nonreversal days represented in Fig. 1, is shown in Fig. 2. The data, further broken down to permit examination of sequential dependencies based on the order of $\mathrm{S}+$ and $\mathrm{S}-$ trials, show no substantial acceleration in rate preceding reinforcement on S+ trials and nothing very much either in the way of sequential effects. The close agreement between the present data and those generated by the discrete-trials/fixed-ratio procedure also strongly suggests that temporal discrimination is not a matter of concern.

\section{SAMPLE DATA FOR GOLDFISH}

\section{Method}

The Ss were $1210-\mathrm{cm}$ goldfish maintained in individual 15 -liter tanks on a $24-h$ feeding schedule. The animals were

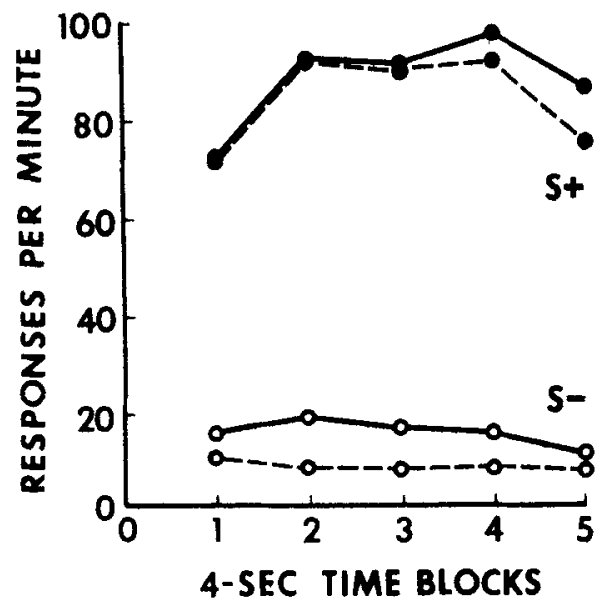

Fig. 2. Changes in rate of response during $20-\mathrm{sec}$ presentations of $S+$ and $S-$ on one of the nonreversal days represented in Fig. 1. The solid lines are for trials preceded by a trial with the same stimulus, while the broken lines are for trials preceded by a trial with the opposite stimulus.

trained in their living tanks, which were arranged on an automatic table. The table could be rotated to bring each tank in turn into the training position, at which the motor-driven manipulandum could be lowered into it. The manipulandum was a translucent Plexiglas target $(4 \mathrm{~cm}$ in diam) illuminated from behind with colored light and attached by a thin rod to a crystal phonograph cartridge, the output of which was used to drive a relay. At the center of the target was a small foodcup, into which could be pumped the liquid food used as reward. For details of this system, see Woodard and Bitterman (1974). The training method was the same as for the pigeons, except that there was a $10-\mathrm{sec}$ reinforcement period during which the target (which served as the feeder as well as the manipulandum) was illuminated with the $\mathrm{S}+$ color. The first response after the $20-\mathrm{sec}$ lock-up on S+ trials produced a drop of food and started the reinforcement timer; each subsequent response during the reinforcement period produced another drop of food.

\section{Results and Discussion}

The asymptotic performance of goldfish trained in a series of 2-day red-green (Wratten No. 25 and No. 58) reversals is shown in Fig. 3. The main difference between
Fig. 3. Asymptotic 2-day within-sessions reversal learning in goldfish.

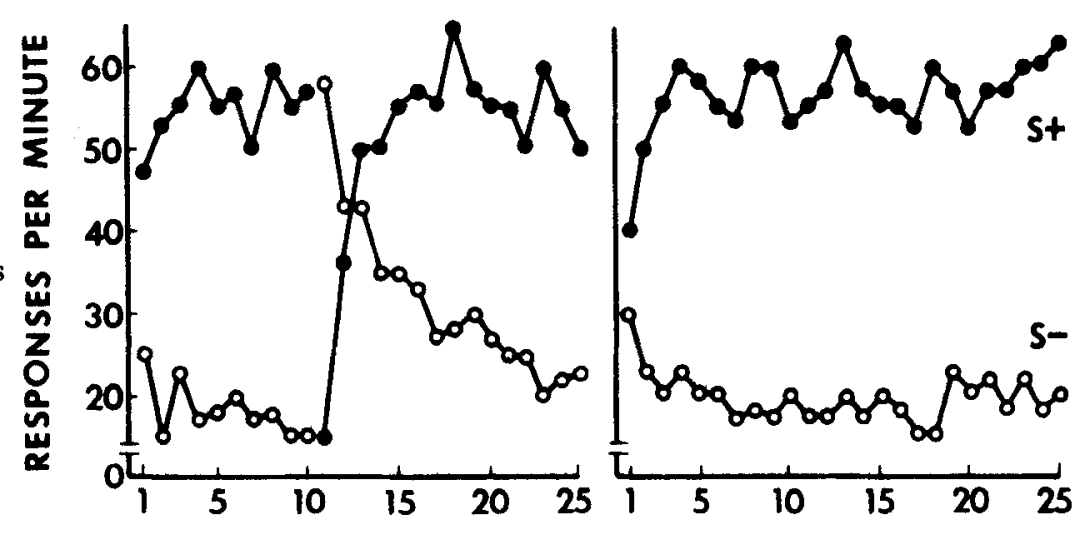


DAY 1

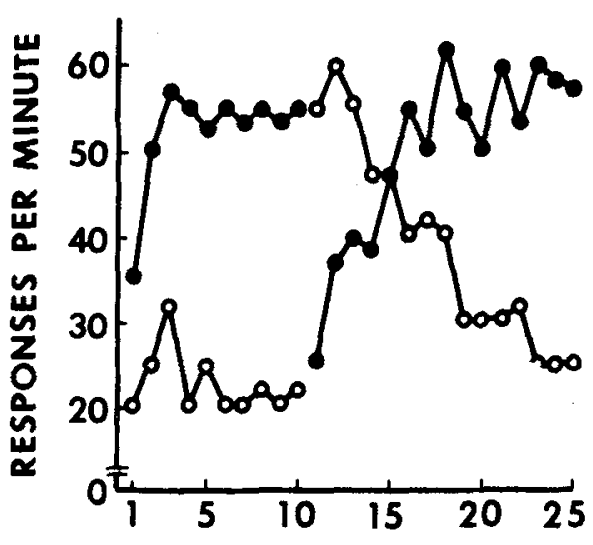

DAY 2

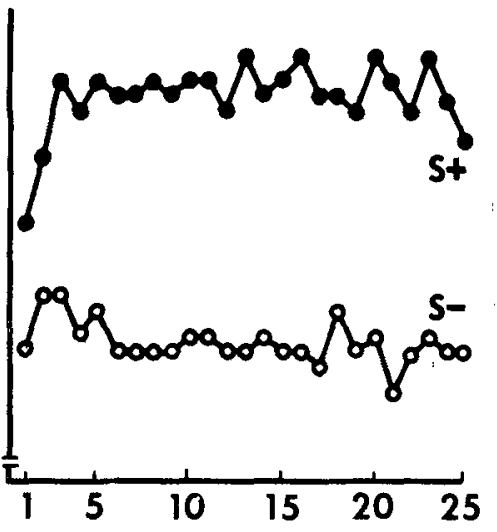

Fig. 4. Asymptotic 2-day within-sessions reversal learning in goldfish. The conditions are the same as those which provided the data of Fig. 3, except that the S-penalty was omitted.

\section{TRIALS}

the procedure employed here and that which yielded the pigeon performance shown in Fig. 1 is that the positive and negative stimuli were reversed within sessions-after the 20th trial on Day 1. The general shape of these curves is very much the same as those obtained with the traditional free-operant/variable-interval procedure by Tennant and Bitterman (1973, cf. their Fig. 5), but the present curves are based on daily sessions about half as long and permit a much finger-grain analysis of changes in behavior, showing separately the effect of each reinforced and nonreinforced experience with the stimuli.

Since the use of the $S$ - penalty may be criticized on the ground that it permits variability in the amount of $S$ - experience across Ss, the effect of the penalty was investigated by removing it (responses to $S-$ after the 20-sec lock-up no longer reset the penalty timer) for a series of five reversals immediately following those which provided the data plotted in Fig. 3. Pooled performance curves for the last four of these five reversals is shown in Fig. 4. The curves suggest that the penalty produces a somewhat more precipitous decline in S- responding and a somewhat lower terminal level; St responding is largely unaffected. Unfortunately, we have no comparable data for naive Ss trained from the outset without the penalty and therefore no indication of its worth early in training. The present comparison does indicate, however, that whatever the effect of the penalty in earlier reversals, it may be removed at a later stage of training without substantial disruption in performance.

The asymptotic performance of goldfish trained (with the $S$ - penalty) in a series of daily red-green reversals in which there were 20 trials per reversal is shown in Fig. 5 . These curves have very much the same appearance as the daily reversal curves obtained by Tennant and Bitterman (1973, cf. their Fig. 3) under conventional free-operant/variable-interval conditions, despite the fact that the present curves are based on performance in 20 20-sec rather than 203 -min trials-a substantial saving in time.

\section{REFERENCES}

Behrend, E. R., Bauman, B. A., \& Bitterman, M. E. Probability-discrimination in the fish. American Joumal of Psychology, 1965, 78, 83-89.

Graf, V., Bullock, D. H., \& Bitterman, M. E. Further experiments on probability-matching in the pigeon. Journal of the Experimental Analysis of Behavior, 1964, 7, 151-157.

Schoel, W. M., Davis, J., \& Bitterman, M. E. Adventitious reinforcement in free-operant discrimination. Behavior Research Methods \& Instrumentation, 1971, 3, 8-9.

Tennant, W. A., \& Bitterman, M. E. Asymptotic free-operant discrimination reversal in the goldfish. Journal of Comparative \& Physiological Psychology, 1973, 82, 130-136.

Woodard, W. T., \& Bitterman. M. E. Improved techniques for the measurement of consummatory behavior in fishes. Behavior Research Methods \& Instrumentation, 1974, 6, 321-324.

woodard, W. T., Schoel, W. M., \& Bitterman, M. E. Keversal learning with singly-presented stimuli in pigeons and goldfish. Journal of Comparative \& Physiological Psychology, 1971, 76, 460-477.

(Received for publication February 23, 1974;

Fig. 5. Asymptotic daily reversal learning in goldfish. revision received March 27, 1974.) 